\title{
COMMUTATORS FOR MULTIPLIERS ON BESOV DUNKL SPACES
}

\author{
SALLAM HASSANI AND MOHAMED SIFI
}

Abstract. In this paper, we first study the boundedness properties of the Dunkl multiplier of the interval $[a, b]$ associated with the reflection group $\mathbb{Z}_{2}$. Next, we prove that the commutator $\left[T, T_{\mu}\right]$ is bounded on the Besov Dunkl spaces $B D_{p}^{\sigma, q}$, if $T$ is a bounded linear operator on $B D_{p}^{\sigma_{j}, q_{j}}\left(j=0,1\right.$ and $\left.0<\sigma_{1}<\sigma<\sigma_{0}\right)$ and $T_{\mu}$ is a dyadic admissible multiplier. These results are obtained for the multi-dimensional Dunkl transform associated to the reflection group $\mathbb{Z}_{2}^{d}$.

Mathematics subject classification (2010): Primary 33C52; secondary 42B10, 43A32, 33C80, 22E30. Keywords and phrases: Dunkl transform, Dunkl multipliers, commutators, Besov Dunkl spaces.

\section{REFERENCES}

[1] C. Abdelkefi, J. Ph. Anker, F. Sassi And M. Sifi, Besov type spaces on $\mathbb{R}^{d}$ and integrability for the Dunkl transform, Symmetry, Integrability and Geometry: Methods and Applications 5 (2009), Paper 019, 15 pages.

[2] C. Bennett And R. Sharpley, Interpolation of Operators, Academic Press, New York, 1988.

[3] J. BerGH AND J. LÖFströM, Interpolation Spaces, An Introduction, Springer, Berlin, Heidelberg, New York, 1976.

[4] J. J. Betancor, Ò. Ciaurri And J. L. VARona, The multiplier of the interval $[-1,1]$ for the Dunkl transform on the real line, J. Funct. Anal. 242 (2007), 327-336.

[5] J. Cerdà, N. Ya. KRugljaK And J. Martín, Commutators for approximation spaces and Marcinkiewicz type multipliers, J. Approx. Theory 100 (1999), 251-265.

[6] J. CERDÀ AND J. MARTín, Commutators for Fourier multipliers on Besov spaces, J. Approx. Theory 129 (2004), 119-128.

[7] M. CWikel, B. Jawerth And M. Milman, The domain spaces of quasilogarithmic operators, Trans. Amer. Mat. Soc. 317 (1989), 599-609.

[8] M. Cwikel, B. Jawerth, M. Milman and R. Rochberg, Differential estimates and commutators in interpolation theory, Analysis at Urbana, Vol. II (Urbana, IL, 1986-1987), 170-220, London Math. Soc. Lecture Note Ser., 138, Cambridge Univ. Press, Cambridge, 1989.

[9] M. F. E. DE JEU, The Dunkl transform, Invent. Math. 113, 1 (1993), 147-162.

[10] C. F. DunKL, Differential-difference operators associated to reflection groups, Trans. Amer. Math. Soc. 311, 1 (1989), 167-183.

[11] C. F. DunkL, Hankel transforms associated to finite reflection groups, in: Proc. of special session on hypergeometric functions on domains of positivity, Jack polynomials and applications, Proceeding, Tampa 1991, Contemporary Mathematics 138 (1992), 123-138.

[12] B. JAWERTH, R. ROCHBERG AND G. WeISS, Commutators and other second order estimates in real interpolation theory, Ark. Mat. 24 (1986), 191-219.

[13] A. NOWAK AND K. STEMPAK, Relating transplantation and multipliers for Dunkl and Hankel transforms, Math. Nachr. 11 (2008), 1604-1611.

[14] J. Peetre And G. Sparr, Interpolation of Normed Abelian Groups, Ann. Math. Pura Appl. 92 (1972), 217-262.

[15] R. Rochberg And G. Weiss, Derivatives of analytic families of Banach spaces, Ann. of Math. 118 (1983), 315-347.

[16] M. RöSLER, Bessel-type signed hypergroups on $\mathbb{R}$, Probability measures on groups and related structures, XI (Oberwolfach, 1994), 292-304, World Sci., Publ., River Edge, NJ, 1995. 
[17] M. RÖSLER, Dunkl operators: theory and applications, In Orthogonal polynomials and special functions (Leuven 2002), 1817 of Lectures Notes in Math. (2003), Springer Berlin, 93-135.

[18] K. TRIMÈche, Paley-Wiener theorems for the Dunkl transform and Dunkl translation operators, Integral Transforms Spec. Funct. 13, 1 (2002), 17-38.

[19] G. N. Watson, A Treatise on the theory of Bessel Functions, Cambridge University Press, Cambridge, England (1944).

[20] Y. XU, Orthogonal polynomials for a family of product weight functions on the spheres, Canad. J. Math. 49 (1997), 175-192. 